\title{
Singular 'they' and novel pronouns: gender-neutral, nonbinary, or both?
}

\author{
Evan D. Bradley, Julia Salkind, Ally Moore \& Sofi Teitsort*
}

\begin{abstract}
Pronouns have recently become a highly visible aspect of the LGBTQ+ movement among the general public and in linguistics. We investigated whether singular, specific 'they' and the novel singular gender-neutral pronoun 'ze' are interpreted as gender-neutral (slient on gender and the gender binary) or or referring specifically to referents of non-binary gender. Participants read descriptions of scholarship applicants, and guessed which photo matched the applicant they read about from an array of male, female, and non-binary subjects. Results suggest that 'they' is interpreted as gender-neutral, including non-binary/gender-nonconforming referents. 'Ze' does not appear to be recognized by enough English speakers to determine a definitive interpretation.
\end{abstract}

Keywords. gender; pronouns; comprehension; semantics; sociolinguistics; LGBTQ+.

1. Introduction. English lacks a standard gender-neutral singular third-person personal pronoun, but speakers need to refer to non-gendered antedendents - for clarity of mearning, to avoid sexism or bias and to be more inclusive, or because the gender of the referent is unknown or doesn't fit the female/male binary and the speaker wishes to accurately refer to individuals according to their personal preference. Speakers have two primary options when the existing gendered pronouns in English don't suit their intended meaning: to shift the usage of existing words or to coin new ones. For the former, singular 'they' has a long history in English (Balhorn 2004), and is very common in speech (LaScotte 2016). This may stem from the peculiar reference properties of plural pronouns (Borthen 2010). However, this usage has often been criticized based on the claim that 'they' can only be plural (Bodine 1975). New pronouns have been proposed, but none have achieved widespread use (Barron 2010). While most English speakers accept generic singular 'they' (1), ratings diverge on specific uses (2); further, those who reject (2) endorse prescriptivist linguistic views and more binary concepts of gender (Bradley et al. 2017; Bradley 2018), consistent with an analysis by Bjorkman (2017) that specific use (2) is driven by changes in socio-pragmatic conventions and syntactic features.

(1) If a student $\mathrm{i}_{\mathrm{i}}$ asks for help, I try to help them $\mathrm{m}_{\mathrm{i}}$.

(2) When the student ${ }_{i}$ had a problem, they ${ }_{i}$ called me.

Swedish has had success adding a gender-neutral pronoun ('hen') (Sendén et al. 2015), which Lindqvist et al. (2018) found reduces male interpretive bias compared to phrases ('the applicant'), leading to claims that novel pronouns are a better way to achieve gender-neutrality than shifting forms like English 'they'. However, evidence for male bias of singular 'they' may be outdated given recent usage changes. Further, the distinction between gender-neutral (silent on gender) vs. non-binary (referring to gender-nonconforming referents) pronouns is underexplored. Lindqvist et al. (2018) included images only of gender-conforming men and women.

\footnotetext{
${ }^{*}$ We are grateful for additional assistance from Max Schmid and Hannah Lombardo. Corresponding Author: Evan D. Bradley, Penn State Brandywine (evan.d.bradley@psu.edu).
} 
Such comparisons across languages are difficult, because no direct equivalent of singular 'they' was tested in Swedish, and different patterns of results may result from linguistic as well as social differences between Sweden and English-speaking locales such as the United States.

We examined how English speakers interpret singular specific 'they' and other pronouns and the effect of context. The goal of the study was not to determine which strategy is "better"; each faces different challenges, but both forms exist in use and as individual preferences, which should be respected. Understanding the use and meaning of both forms is key information for language reformers who wish to make arguments informed by evidence about the viability of such forms.

We hypothesized that the battle on the grammaticality of generic singular 'they' in English is all but won, so specific 'they' may not be far behind. Therefore, we expect that for those who use it, singular 'they' is gender-neutral, and therefore leaves any bias (or lack thereof) intact. 'Hen', though intended to be neutral, may (because of novelty and its association with LGBTQ+ rights) specify nonbinary (non-female, non-male) gender. Specifically, we expected that specific 'they' will show equal distribution among possible referents, whether or not nonbinary referents are available, while novel pronouns will show a preference for nonbinary referents when they are available.

2. Experiment. Procedures were based on those used by Lindqvist et al. (2018), in which participants read descriptions of individuals, and then later chose photos which they thought represented the person they had read about. English-speaking participants ( $N=123$; 77 women, 41 men, 4 nonbinary, 1 agender; age 18-101, $m=21.6$; predominately from the United States) participated in an online study.

2.1 PROMPTS. Participants read ten descriptions of scholarship applicants, in the following format:

Student A is a first-year student majoring in Biology. They have a cumulative GPA of 4.0. They would like to pursue a career in Environmental Technology. They are president of the Entrepreneurial League, and they started a composting initiative on campus last year. In their spare time, they enjoy backpacking and camping.

Applicants were referred to as 'they/he/she/ze/the student' (twice each). Major, GPA, and other characteristics were randomized and pronouns were counterbalanced across texts/participants to avoid gendered associations.

2.2 Рното SElection. After reading the descriptions, participants rated students academically and selected from an array of photos which person they thought was the one they had read about them. Some participants saw two feminine- and two masculine-appearing photos (Binary Condition); Others also saw two photos of subjects who did not appear to be clearly female nor male (Nonbinary Condition). Photos arrays were created using stock photos (Figure 1) which were rated by a separate sample of participants $(N=50)$ on femininity and masculininity (independent 10-point scales). Based on these ratings, photos were grouped into feminine, masculine, and nonbinary categories: feminine/masculine photos showed clear gen- 
der classification based on the difference between feminine ratings $(>6)$; non-binary photos had near-equal masculinity-femininity ratings (difference $<3$ ).


Figure 1: Examples of photos used for selection.

2.3 RESUlts. Results were analysed using Chi-squared tests to determine if the distribution of responses differed from an equal distribution of feminine, masculine, and nonbinary responses. For the phrasal circumlocution 'the student,' response distributions did not differ significanty from an equal distribution in either condition $\left(\chi^{2} p>.05\right)$ (Figure 2).
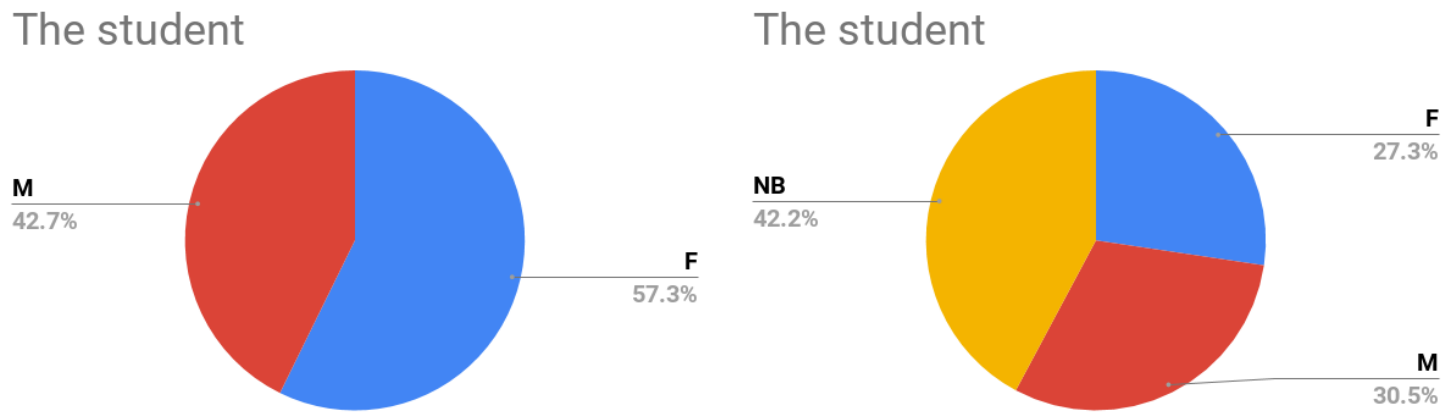

Figure 2: Picture selection for 'the student.'

2.4 GENDERED PRONOUNS. For gendered pronouns 'she' and 'he', feminine and masculine photos, respectively, were the most frequently chosen in both conditions, and distributions differed significantly from an equal distribution $\left(\chi^{2} p<.001\right)$ (Figure 3 ).

An interesting difference between these pronouns emerged in the nonbinary condition; although the matching gendered images were still the most frequently chosen, masculine photos were selected $71.9 \%$ of the time for 'he', while feminine photos were selected $46.9 \%$ of the time for 'she,' with nonbinary photos selected $41.4 \%$ of the time for 'she'(Figure 2).

2.5 SINGULAR THEY. For singular specific 'they,' response distributions did not differ significanty from an equal distribution in either condition $\left(\chi^{2} p>.05\right)$ (Figure 4). 

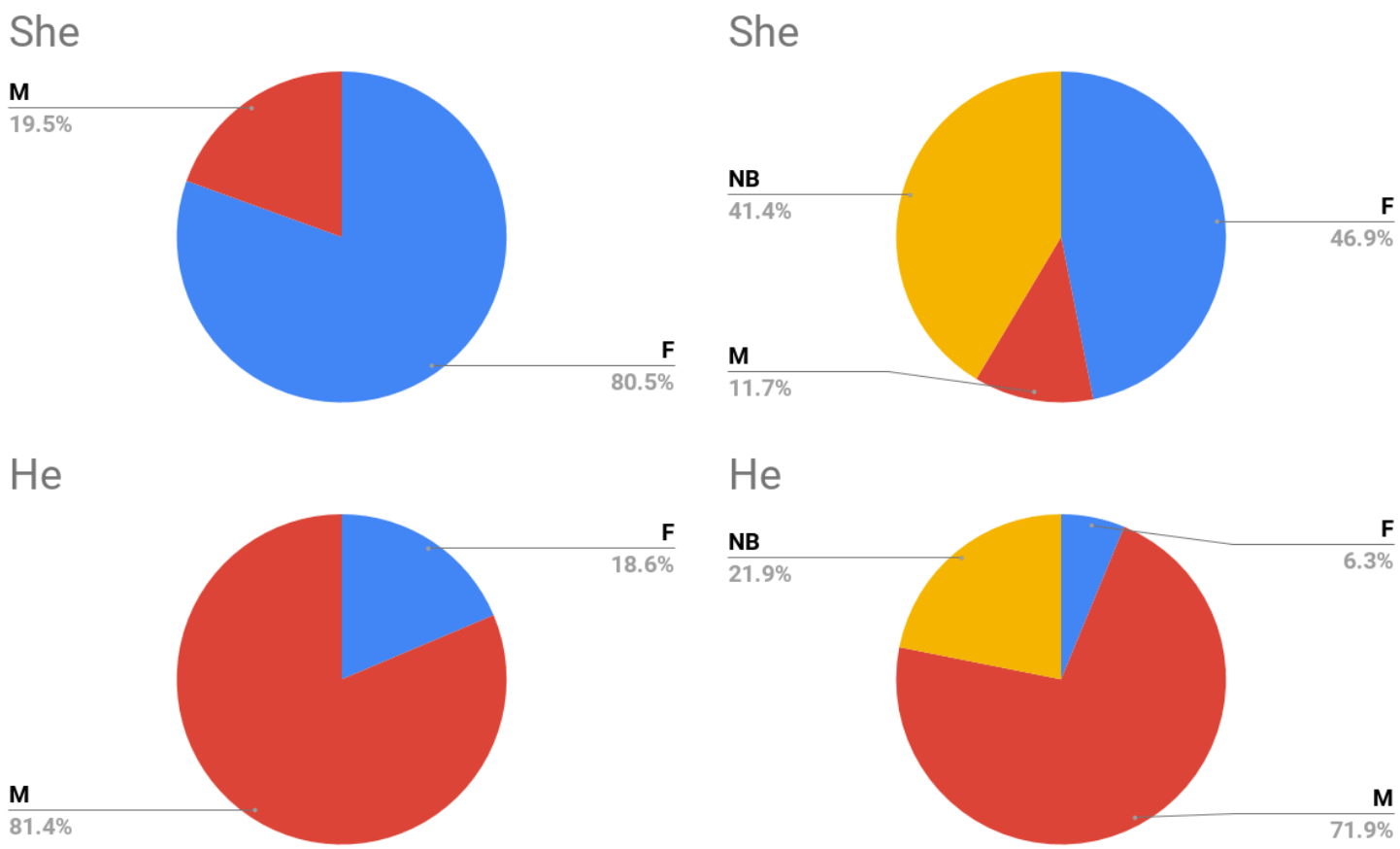

Figure 3: Picture selection for 'she' and 'he.'

2.6 ZE. In both the Binary and Nonbinary conditions, 'ze' showed a strong bias toward masculine photo selection, and response distrbutions differed from equal distributions $\left(\chi^{2} p<.001\right)$ (Figure 5).

2.7 FAMILIARITY. We also asked participants whether they knew anyone who preferred the pronoun 'they' or some other non-standard pronoun.

Whether or not a participant knew someone who used 'they' (47.5\% reported that they did) did not affect how often they chose each kind of photo-both distributions were genderneutral (equal feminine, masculine, and nonbinary).

However, familiarity with someone who used a novel pronoun like 'ze' (15.1\% reported that they did) did have an apparent effect on photo selection. Those who knew someone showed a gender-neutral photo selection (equal feminine, masculine, and nonbinary) while those who didn't showed the strong male bias (Figure 6).

3. Discussion. Results indicate 'they' is a viable option for a gender-neutral and non-binary pronoun in English, contrary to previous results (Lindqvist et al. 2018). 'They' appeared to be gender-netural, which could represent a difference between the younger and perhaps more progressive sample of participants in our experiment compared to previous studies (Lindqvist, p.c.).

Even those who don't know someone who goes by 'they' interpret it as gender-neutral, suggesting it may be a "naturally occuring" option for gender-neutrality and nonbinariness. In comparison to our grammaticality data (Bradley 2018), this suggests that acceptance in grammaticality judgments may even lag interpretation. 

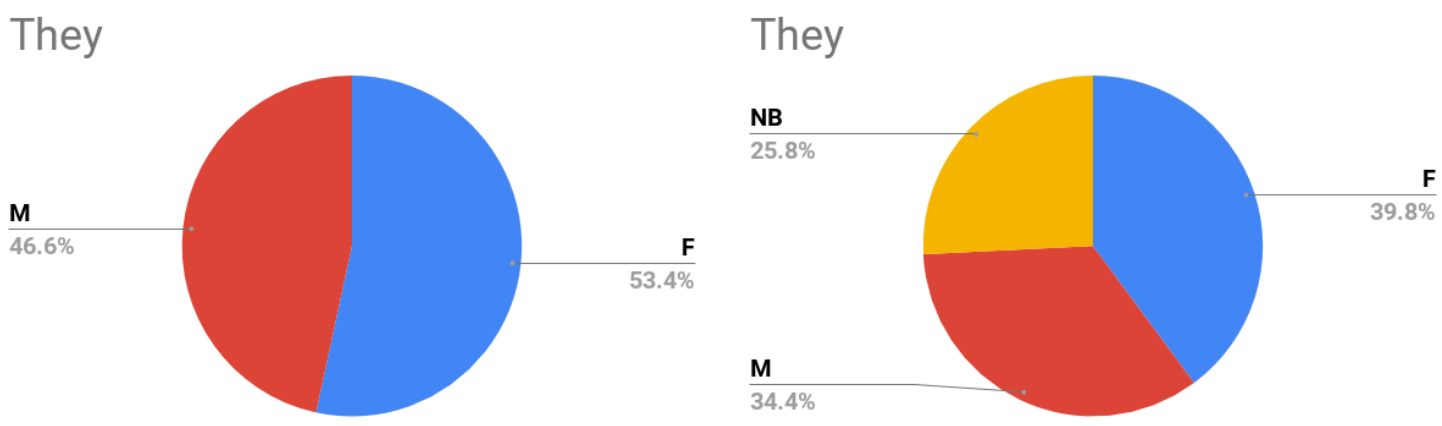

Figure 4: Picture selection for 'they.'
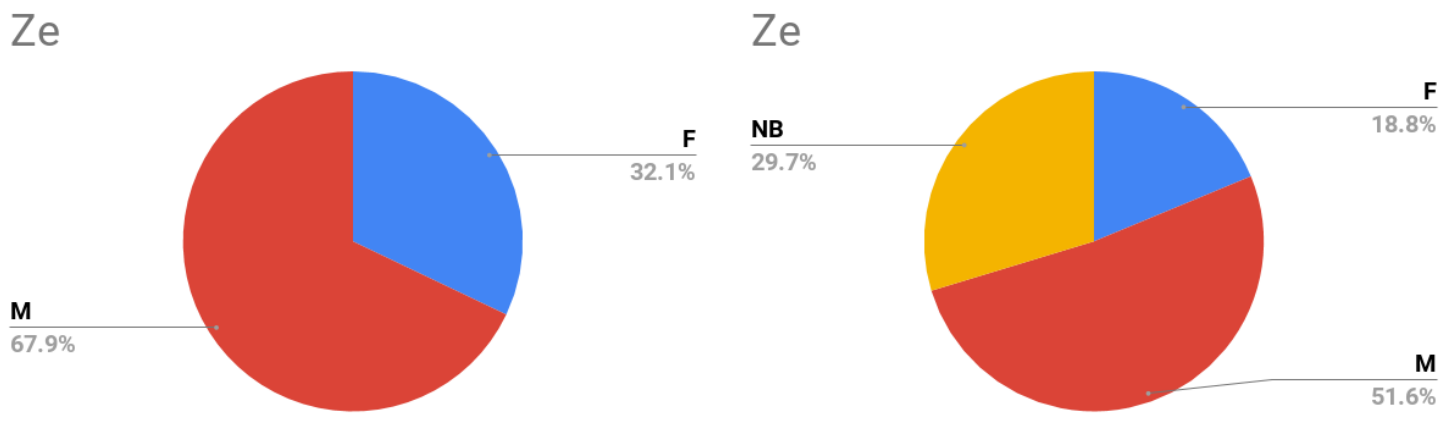

Figure 5: Picture selection for 'they.'

Circumlocution is conspicuous as avoidance of gender. 'The student' had the highest rate of nonbinary interpretation, which may indicate that repeating full NPs frequently is highly unnatural, which drew participants' attention to avoidance of pronouns/gender.

We suspect that those unfamiliar with 'ze' read it as a typo for 'he,' and this is a limitation of the text-based paradigm used in this study. Those who are familiar familiar with it interpret it as gender-neutral, but not non-binary (referring specifically to non-female, non-male individuals). This suggests that wider awareness could lead to rapid acceptance and use.

These results set the stage for future investigations of how pronouns carry gender-neutral and nonbinary meaning. In particular, we would like to know how and when consensus will emerge in English as to how to refer to individuals without specifying their gender. Further, this interpretive data can help us determine how the social factors we've identified (Bradley et al. 2017; Bradley 2018) influence speakers progression from understanding the meanings of these pronouns to using them in their own speech. 
I do not know someone...

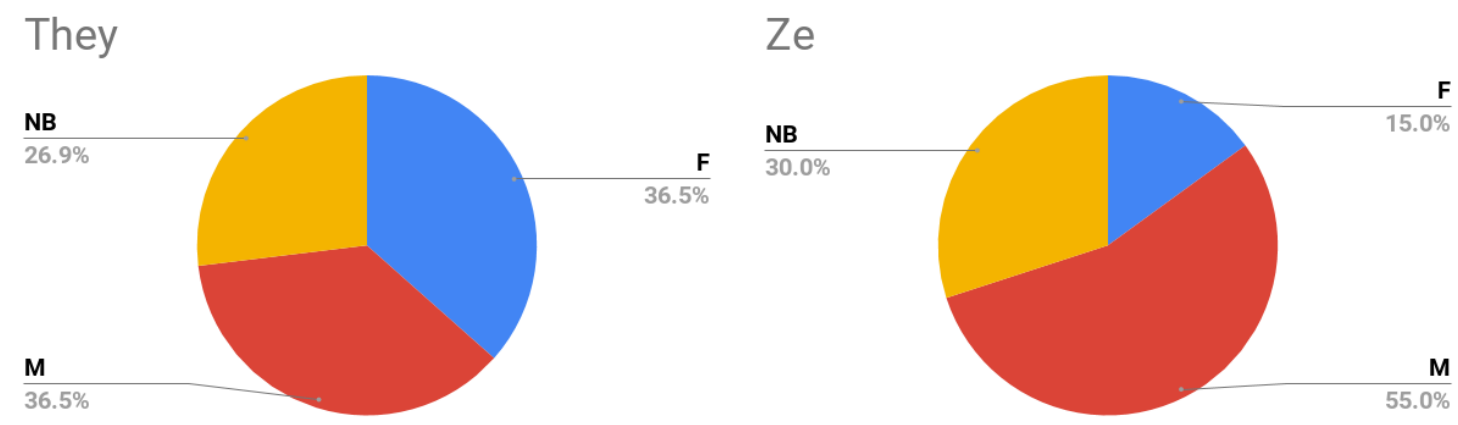

I know someone...
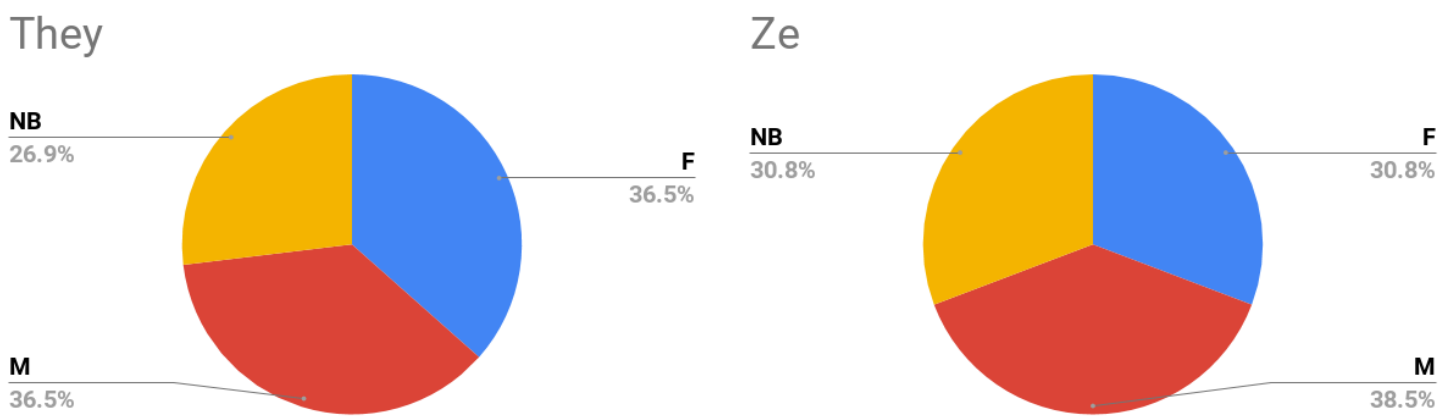

Figure 6: Picture selection by acquaintance with pronoun.

\section{References}

Balhorn, Mark. 2004. The rise of epicene they. Journal of English Linguistics 32(2). 79-104. https://doi.org/10.1177/0075424204265824.

Barron, Dennis. 2010. The gender-neutral pronoun: 150 years later, still an epic fail. https://blog.oup.com/2010/08/gender-neutral-pronoun/.

Bjorkman, Bronwyn M. 2017. Singular they and the syntactic representation of gender in English. Glossa 2(1). https://doi.org/10.5334/gjgl.374.

Bodine, Ann. 1975. Androcentrism in prescriptive grammar: singular 'they', sex-indefinite 'he', and 'he or she'. Language in Society 4(2). 129-146. https://doi.org/10.1017/S0047404500004607.

Borthen, Kaja. 2010. On how we interpret plural pronouns. Journal of Pragmatics 42(7). 1799-1815. https://doi.org/10.1016/j.pragma.2009.02.008.

Bradley, Evan D. 2018. Singular they: links between grammaticality judgments, prescriptivism, and sexism. In International society for language studies, Waterloo, ON, Canada.

Bradley, Evan D., Maxwell Schmid \& Hannah Lombardo. 2017. Linguistic or gender prescriptivism? judgments of English gender-neutral pronouns. In Nordic conference on language and gender, Akureyri, Iceland. 
LaScotte, Darren K. 2016. Singular they: An empirical study of generic pronoun use. American Speech 91(1). 62-80. https://doi.org/10.1215/00031283-3509469.

Lindqvist, Anna, Emma Aurora Renström \& Marie Gustafsson Sendén. 2018. Reducing a male bias in language? establishing the efficiency of three different gender-fair language strategies. Sex Roles 1-9. https://doi.org/10.1007/s11199-018-0974-9.

Sendén, Marie Gustafsson, Emma A Bäck \& Anna Lindqvist. 2015. Introducing a genderneutral pronoun in a natural gender language: The influence of time on attitudes and behavior. Frontiers in Psychology 6. https://doi.org/10.3389/fpsyg.2015.00893. 